trolyte a method of determining total tin and antimony from their sulfides or chlorides is provided.

3. Ammonium, Sodium and Potassium Tartrates and Tartaric Acid.The results for a completely quantitative deposition of antimony were negative. A certain amount of antimony always remains in solution.

4. Calcium Sulfide.--Of the alkaline earth sulfides, that of calcium was shown to be best suited for the deposition of antimony. The deposits were made most successfully with rotating anodes and were of a brilliant platinum-like appearance under suitable conditions. The possibility of the separation of antimony from tin by the precipitation of the tin as stannic hydroxide and the electrolysis of the antimony from the latter in a solution of calcium sulfide was demonstrated.

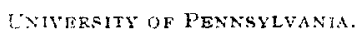

\title{
SOME COLLOIDAL SOLUTIONS DERIVED FROM HYDRATED ALUMINA.
}

By WhliaM B. Bentley and R. PHillips Rosk.

Received August 15, 1915.

It has long been a matter of experience in this laboratory that, in complex solutions used for qualitative analysis, barium is often missed. It has been suspected that the missing barium was precipitated with some of the preceding elements, possibly aluminum. The present research was therefore undertaken for the purpose of ascertaining if barium was brought down from a solution containing aluminum when the latter was precipitated with ammonium hydroxide. Our results indicated an affirmative answer to this question but in attempting to discover the proportion in which these elements were precipitated together, an observation was made which led us to postpone the original problem and take up what appeared to be a more interesting question.

A precipitate of aluminum hydroxide containing some barium was in process of analysis for barium when it was dissolved in acetic acid and acidified with hydrochloric acid previous to the precipitation of barium with sulfuric acid. To our surprise hydrochloric acid gave a gelatinous precipitate resembling somewhat aluminum hydroxide. We at first supposed that this precipitate contained barium but on repeating the experiment with aluminum hydroxide which contained no barium, the same result was obtained. We were unable to find in the literature available anything which threw light on this phenomenon and therefore determined to investigate the reaction and find, if possible, the identity of the precipitated substance. In this object we were only partially successful for our work revealed difficulties which seemed insurmountable.

Naturally our first attempt was to get the substance out of the solution and analyze it. We tried all ordinary filtering media including the densest 
paper, Gooch crucible with thick asbestos mat, and the densest grade of alundum crucible, but none of these would retain more than a small portion of the precipitated matter. It was shaken with barium sulfate in the hope that this substance. would weigh it down and thus make it possible to filter it out but this expedient also failed. It was finally found that by whirling in a centrifugal machine, the gelatinous substance could be separated from the solution. This enabled us to wash out soluble matter, at least partially, but did not help us to get the material in a dry condition and therefore did not make a direct analysis possible. The appearance of the precipitate indicated a colloidal nature and suggested that an hydrosol was formed by the action of acetic acid on the aluminum hydroxide and that this hydrosol was coagulated by the hydrochloric acid added. Many experiments were made in the attempt to determin the nature of the reaction. Various acids were substituted for hydrochloric but only one other, nitric acid, behaved like hydrochloric and produced coagulation. Sulfuric acid not only produced no turbidity itself but cleared the solutions coagulated by hydrochloric or nitric acids. Experiments were also made to see if the acetic acid used as solvent could be replaced by other organic acids. Many of the acids used gave negative results because of the insolubility of the aluminum hydroxide, but formic, propionic and butyric acids behaved like acetic except that the last two were much less effective. That these acids all belong to the same homologous series may or may not be significant.

We next considered the condition of the hydrated alumina which gave best results in the formation of the hydrosol. In this connection we obtained results which seemed to us of interest and we shall accordingly present them in some detail in another paper. In our study of the solution of specimens of hydrated alumina of varying water content, we made the following observations. Using a sample which showed on analysis water corresponding to $\mathrm{Al}_{2} \mathrm{O}_{3} .6 \mathrm{H}_{2} \mathrm{O}$ or $\mathrm{Al}_{2} \mathrm{O}_{3} .7 \mathrm{H}_{2} \mathrm{O}$, the material swelled up and stuck to the sides of the beaker and to the stirring rod. The solution finally obtained was so viscous that air bubbles were held suspended in it for several weeks. Acidified with hydrochloric acid it became a stiff jelly but finally liquefied. The appearance of the material was somewhat like that of the product obtained when sodium silicate is acidified, except of course, in the matter of final liquefaction. By using specimens containing $4^{1 / 2}-6$ molecules of water, solution was readily effected but the solutions were opalescent and viscous and when shaken formed bubbles like soap solutions, the films sometimes appearing even tougher than soap bubbles. On acidification, gelatinization and subsequent liquefaction took place as before. Specimens containing $2^{3 / 4}-4$ molecules of water dissolve readily and form solutions much less opalescent and viscous. These solutions are coagulated by hydrochloric acid but do not form a jelly. 
Probably there is a smaller percentage of coagulum in these cases though the nature of the precipitate makes this difficult, if not impossible, to prove. If the hydrated alumina contained less than $2^{3} / 4$ molecules of water, solution was difficult and was accomplished only by prolonged heating. The solution thus obtained appeared to be normal aluminum acetate, since it would not coagulate as the other solutions had done, on the addition of hydrochloric acid. That this solution was indeed normal aluminum acetate did not seem easy to prove positively, since this substance cannot be isolated from the solution for identification. There is, however, little room for doubt on this point as there does not seem to be anything else which it could well be.

Our general conclusion was that the most favorable condition for the formation of the colloidal solution was that the hydrated alumina should contain a high percentage of water. We also found that if dried specimens were used, that which was dried at the lower temperature was better for the purpose than that which had been dried at a higher temperature even though the water content of the two samples was the same.

Concerning the strength of acetic acid which should be used, we made the following experiments: Equal portions of a specimen of the hydrated oxide containing about four molecules of water were treated with equal volumes of acetic acid varying by tens from $99.8 \%$ acid down to $40 \%$ and by ones down to $\mathrm{x} \%$. It was found that above $40 \%$ acid scarcely any hydrosol was formed, since hydrochloric acid did not produce coagulation. Normal aluminum acetate was the product formed. From $40 \%$ down the amount of hydrosol steadily increased as did the ease with which the material dissolved, until a $4 \%$ acid was reached. With acid below $4 \%$ the material dissolved only partially, the remainder swelling up to an almost transparent mass which after prolonged treatment formed a very opalescent and most easily coagulated solution. For practical purposes we consider about $8 \%$ acetic acid the most favorable strength.

The method of preparation which we adopted as most suitable from all points of view was as follows: the hydrated alumina was precipitated from an alum solution in the usual way, thoroughly washed and dissolved in hydrochloric acid. This solution was precipitated a second time with ammonium hydroxide and washed until free from sulfates and chlorides. Without drying, this product was added to hot acetic acid of about $8 \%$ concentration until no more would dissolve. The solution thus formed was filtered through an alundum crucible which seemed to be the most satisfactory filter for the purpose. In most cases a solution thus prepared could be preserved for a considerable period, but we might add that even this method occasionally failed to give the desired result. Our work indicated that thorough washing of the aluminum hydroxide was of the utmost importance. The presence of any impurity due to incomplete 
washing seemed to hinder the formation of the hydrosol, presumably because such impurities act in the same way as the agents used for coagulation.

\section{Coagulation of the Colloidal Solution.}

As has been stated above, the addition of hydrochloric acid produced a precipitate in the colloidal solution made by the process just described. The same result followed the addition of potassium sulfate and if the solution was submitted to prolonged boiling without the addition of any reagent, coagulation occurred on centrifuging and sometimes by simply allowing the material to stand. The coagulum produced by this latter operation was similar in appearance, and, as will be shown later, it was also similar in behavior to that produced by hydrochloric acid. The coagulum produced by potassium sulfate, however, differed somewhat in appearance and decidedly in behavior, from both of them. It appeared probable that normal aluminum acetate, which was the sole product under some conditions as noted above, was always produced in greater or smaller quantities. Prolonged boiling would, of course, increase the quantity formed and we therefore judged that the coagulation was produced by this substance. To test this supposition we submitted a specimen, which had boiled for a long time, to centrifugation and drew off the clear supernatant liquid. This liquid when tested with ammonium hydroxide showed the presence of aluminum and must have contained normal aluminum acetate.

When it was discovered that we could separate the precipitate from the liquid portion by use of the centrifuge, we supposed that we should be able to determin the nature of the precipitate by washing it free from other matter and then submitting it to analysis. Accordingly, after the separation of the first liquid, we added water for the purpose of washing out the soluble matter. The result was that the whole precipitate dissolved and we were unable to throw it out of solution though the speed of the centrifugal was increased to about 2200 revolutions per minute. Having failed to throw any light on the nature of the coagulum by this experiment, we next tried to accomplish our purpose by determination of the proportion of alumina and acetic acid in the coagulum by indirect means. A colloidal solution was made in the way described above and was then analyzed for alumina and acetic acid. The alumina was determined by precipitating a measured volume of the solution in the usual way with ammonium hydroxide, and weighing the ignited aluminum oxide. A measured volume was also acidified with sulfuric acid and distilled until the liberated acetic acid had passed over. The acetic acid thus distilled was estimated by titration with standard alkali. For convenience all results are calculated on the basis of a liter of solution. The solution was then centrifugated at $\mathrm{I} 800$ to 1900 revolutions per minute. This process 
resulted in separating at least a part of the colloidal material in a mass which adhered to the bottom of the vessel in which the centrifugation had taken place. With a pipette about one-half of the original volume could be withdrawn in the form of a clear liquid. This liquid was then analyzed for alumina and acetic acid using the same methods as before and these results were also calculated into grams per liter. By deducting from the amount of alumina in the original solution that which was found in the clear centrifugated liquid we found the amount which was contained in the precipitate and of course the same was true of the acetic acid. The volunie of solution used for analysis was in each case $10 \mathrm{cc}$, and the results. are here presented in tabular form.

\begin{tabular}{|c|c|c|c|c|c|}
\hline Ni., & Substance deternined. & $\begin{array}{l}\text { Wught } \\
\text { observed. } \\
\text { original. }\end{array}$ & Duplicate. & Sean. & $\begin{array}{l}\text { Calcuiated } \\
\text { weight per } \\
\text { liter. }\end{array}$ \\
\hline I. & $\mathrm{Al}_{2} \mathrm{O}_{3}$ in original solution & 0.0838 & 0.0872 & 0.0855 & 8.550 \\
\hline 2. & $\mathrm{Al}_{2} \mathrm{O}_{3}$ in supernatant liquid & 0.0370 & 0.0375 & 0.03725 & 3.725 \\
\hline 3. & \multicolumn{4}{|c|}{$\mathrm{Al}_{2} \mathrm{O}_{3}$ in colloid from $\mathrm{I}$ liter (by difference of $\mathrm{I}$ and 2 ) } & 4.825 \\
\hline 4. & Acetic acid in original solution & 0.08415 & 0.08415 & 0.08415 & 8.415 \\
\hline 5. & Acetic acid in supernatant liquid & 0.06865 & 0.06866 & 0.06866 & 6.866 \\
\hline 6. & \multicolumn{4}{|c|}{ Acetic acid in precipitate from I liter (difference of 4 and 5 ) } & i. 549 \\
\hline
\end{tabular}

'Thus we see that the ratio of alumina to acetic acid is:

In the original solution 8.55 to 8.415 .

In the precipitated colloid 4.825 to 1.549 .

In the decanted liquid 3.725 to 5.866 .

Calculating from these ratios the amount of acetic acid corresponding to $\mathrm{Al}_{2} \mathrm{O}_{3}$ ( $\mathrm{I} 02.2$ units) we have in the original solution 100.6 units or $\mathrm{I}^{2} / 3+$ molecules acetic acid, in the precipitated colloid $32.8 \mathrm{I}$ units or $1 / 2-$ molecules acetic acid and in the decanted liquid 188.4 units or $3+$ molecules acetic acid. From the nature of the experiment one could scarcely expect figures of great precision; but, as normal aluminum acetate requires six molecules of acetic acid to one of alumina, and this ratio is not reached even in the decanted liquid, we must conclude that our solution consists of a basic acetate mixed more or less with the normal acetate. According to Beilstein (Handbuch der Organischen Chemie) there is but one soluble basic aluminum acetate $\mathrm{Al}_{2}\left(\mathrm{C}_{2} \mathrm{H}_{3} \mathrm{O}_{2}\right)_{4} \mathrm{O}$ and this should therefore be contained in the decanted liquid. As to the precipitate, the formula which is apparently nearest in correspondence with the above figure is $\left(\mathrm{AlOC}_{2}\right.$ $\left.\mathrm{H}_{3} \mathrm{O}_{2}\right)_{2}\left(\mathrm{Al}_{2} \mathrm{O}_{3}\right)_{3}$. This would yield alumina and acetic acid in the ratio $3.407:$ I while our ratio as determined above is $3.116: 1$. An obvious suggestion as to the identity of the precipitate would be that it was simply alumina holding back some acetic acid in the form of normal aluminum acetate or in some other form and that the amount of acetic acid thus retained was accidental rather than definit. This view we reject because the precipitate dissolved when treated with a considerable quantity of water as described above. It seems impossible or at least unlikely that 
an indefinit mixture should dissolve in this way. Our interpretation was that so long as there was present in the supernatant liquid a certain quantity of normal aluminum acetate the precipitate remained insoluble or at least capable of being thrown out of solution by centrifugation but when the dilution of this reagent had been sufficiently increased, the precipitate again passed into the original colloidal solution. In confirmation of this view we added to a precipitate thrown out by centrifugation, as described above, a very small quantity of water. After stirring the materials together it was possible to throw the precipitate out by centrifugation. This operation was possible several times, if the amount of water added was small enough, but in any event after several repetitions of this treatment the substance went into solution and could not be thrown out again, though it was possible to form a precipitate by adding potassium sulfate. We have just stated that we believe that the reagent which made possible the separation of the precipitate, the substance which was removed by the washing process just described, was normal aluminum acetate. It must be this compound or acetic acid for the only substances present in the solution were alumina, acetic acid and the products of their interaction. We have repeatedly tried to precipitate these solutions by adding quantities of acetic acid but always without success. We take it then that normal aluminum acetate precipitates this basic acetate in the same way that common salt precipitates other sodium salts like soap. The real coagulating agent is then the aluminum ions, the acetate ions or both.

\section{Hydrochloric Acid as Coagulating Agent.}

The coagulation with hydrochloric acid was studied quantitatively by adding to equal volumes of the colloidal solution, varying amounts of twice normal acid and making up to a standard volume with water. By centrifugating, a clear solution was obtained and this was analyzed for alumina. By subtracting this quantity from that known to have been in the solution used, we determined the percentage of the alumina precipitated in the experiment. Twenty-five cc. of the solution were taken in each experiment and addition of water and $2 / N$ hydrochloric acid were made as follows:

$\begin{array}{cccc}\text { No. } & \text { Water in cc. } & 2 N \mathrm{HCl} \text { in cc. } & \begin{array}{c}\text { Per cent. } \mathrm{Al}_{2} \mathrm{O}_{3} \\ \text { coagulated. }\end{array} \\ 1 & 18 & 2 & \text { None } \\ 2 & 16 & 4 & \text { None } \\ 3 & 14 & 6 & 54.7 \mathrm{I} \\ 4 & 12 & 8 & 64.02 \\ 5 & 10 & 10 & 65.63 \\ 6 & 6 & 14 & 70.08 \\ 7 & 2 & 18 & 91.08\end{array}$

It thus appears that in order to produce coagulation of this solution enough hydrochloric acid must be added to make the solution about N/5 
in this acid. If the solution was made $0.8 \mathrm{~N}$ for hydrochloric acid over $90 \%$ of the total alumina appeared in the coagulum.

In our earlier work with the coagulum produced by hydrochloric acid we attempted to purify the precipitate by dialysis. After the process had continued for some time the precipitate went into solution. This was the first indication which we had that in order to keep these precipitates in insoluble form, the precipitating agent must be present. When the precipitate was centrifuged and washed it behaved exactly as has already been described in the other coagulation-it dissolved if much water was used. From this solution a coagulum was thrown down by potassium sulfate as before. The following experiment was undertaken in order to throw light on the identity of the precipitate with hydrochloric acid which we at first supposed to be a basic chloride acetate of aluminum. Hydrochloric acid was added to the solution until coagulation occurred. The solution was then centrifuged, the supernatant liquid decanted and the coagulum washed with water containing enough hydrochloric acid to prevent resolution and once with a small quantity of pure water. Enough hydrochloric acid remained in the material to combine with all the alumina and thus liberate any acetic acid present. It was now heated to $100^{\circ}$ conducting all fumes evolved through potassium hydroxide. Our results showed that there was no acetic acid in the fumes evolved and none in the residue left on drying. The behavior of the material during treatment was interesting. On first heating it liquefied forming a solution which we afterwards proved to be colloidal by coagulating it with potassium sulfate. It then reprecipitated and finally dissolved a second time. The first liquefaction must have been due to the increase of temperature and the reprecipitation to the concentration of the hydrochloric by evaporation. The second liquefaction must have been due to the formation of normal aluminum chloride as this substance crystallized out of the evaporating residue. As has been stated, no acetic acid could be found in the absorption apparatus nor in the residue and it would therefore appear that our original colloidal solution consisting of a basic aluminum acetate was probably converted by the hydrochloric acid to a basic chloride, also colloidal, and this by increasing the concentration of chlorine ions through addition of more acid was precipitated. The basic acetate was not precipitated by acetic acid because of the small concentration of acetic acid ions in that acid. We have, however, been able to coagulate the solution by adding sodium acetate and the coagulum thus formed dissolved in water and was capable of reprecipitation by potassium sulfate. We have also been able to secure the same result by adding aluminum sulfate, thus indicating that ions of aluminum were also capable of producing the precipitation of this colloid. We also tried the effect of aluminum chloride and nitrate with the surprising result that they not only did not 
coagulate the solution, but after they had been added potassium sulfate would no longer produce coagulation. The only conclusion possible seems to be that these reagents in some way acted to convert the colloidal into a true solution. In this connection we might recall that nitric as well as hydrochloric acid precipitated the colloidal solution and thus led us to believe that a colloidal basic nitrate as well as chloride was capable of formation. We have no evidence of the formation of a colloidal basic sulfate. Lack of time prevents us investigating further these results with aluminum chloride and nitrate and we therefore mention them with some caution. We also attempted to form the basic chloride by adding hydrochloric acid in quantity insufficient to produce coagulation and then precipitate by adding chlorine ions in the form of sodium chloride. The result was what we expected, for a precipitate was produced which dissolved on adding more water. From this solution a precipitate was thrown down by addition of potassium sulfate. These results seem to us to point very clearly to the formation of ions in our colloidal solutions.

\section{Coagulation by Potassium Sulfate.}

It has been several times stated that potassium sulfate precipitates these colloidal solutions. The substance precipitated by this reagent is, however, different from that formed by either of the other precipitants investigated, for this coagulum will not redissolve in water, even though it is washed repeatedly. It has appeared to us that this precipitation is like that which usually occurs when a colloidal solution is precipitated by an electrolyte.

On several occasions it was noticed that a web structure appeared in the hydrogel after coagulating with potassium sulfate and centrifugating. This web was of denser substance than the surrounding medium. On drying and examining with the microscope crystals of potassium sulfate were found, thus indicating the web as the result of absorption of the crystalloid potassium sulfate by the precipitated colloid. This web structure was difficult to obtain and was of course entirely destroyed by drying.

\section{General Considerations.}

We have repeatedly asserted our belief that the original solution contained colloidal basic acetate and not colloidal alumina. The following experiment confirms this position: Specimens of freshly precipitated aluminum hydroxide were boiled with water for long periods without any formation of colloidal solution. We next tried heating with water to $140^{\circ}$ under pressure. The heating was continued for several days but the results were negative. According to Poschel ${ }^{1}$ colloidal silicic acid has been prepared in this way and if our colloid had been simply hydrated alumina

${ }^{1}$ Chemistry of Colloids. 
it seems probable that some, at least, would have been formed by this treatment.

\section{Ultramicroscopic Examination.}

To identify our solution more positively as colloidal we made use of the Cardioid Ultramicroscope manufactured by Zeiss and described by him in a publication designated as Micro 306 . We may say that the cardioid condenser which is the essential feature of the apparatus condenses a powerful beam of light in the solution under examination in such a way that it can find its way into the microscope only by being reflected from the particles of substance colloidally dissolved in the solution. Of course the utmost care must be taken to prevent the presence of dust or other solid particles which might also reflect the light into the microscope. However, a little experience will enable the operator to distinguish between particles of colloidally dissolved substance and these foreign particles. As light reaches the microscope only by reflection it is clear that the field will be dark except where it is illuminated by reflection from these particles and the effect is very similar to that of stars in the sky on a very dark clear night. Our colloidal solution appeared full of shining particles of all sizes. 'The smaller particles moved with a whirling motion, at times stopping in one spot and whirling, then dashing off to another place to repeat the process. The larger particles were of irregular size and shape and did not move. The particles of intermediate size were of various shapes and moved slowly with the same motion as the smaller ones. Sometimes two of the smaller particles would collide and coalesce without stopping their motion. The smaller particles seemed perfectly round and whirled with an exceedingly rapid motion while at the same time they were constantly changing their position. The smallest particles visible were only seen darting across the field from side to side. That the particles move with different rates according to size is of course exactly what one would expect. It may be noticed, however, that although the larger particles do not move, still they do not settle out of solution. The solution used in this work had been kept for over a month and so far as one could see was perfectly homogeneous. It differed from an ordinary solution only by being slightly opalescent. Many attempts were made to map the path of the particles and to compare them with the paths of other colloidal particles as mapped by other investigators but we could not secure any satisfactory results.

The addition of potassium sulfate stopped all motion, as it coagulated the solution. Instead of the particles appearing singly they then appeared in groups. Sometimes the aggregations would be large enough to cover nearly a third of the field. In this case they sparkled with many colors, caused by the prismatic effect of their membrane, until they were very 
hard to observe. When the conditions were favorable, however, it was possible to find a field full of aggregations made up of a few particles only.

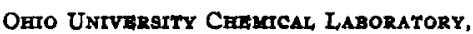

ATrms, Omo.

\section{THE CONSTITUTION OF ALUMINATES. ${ }^{1}$}

Bq WrLtax BLox.

Received August 8, 1913.

In a recent article by Mahin, Ingraham and Stewart upon the constitution of aluminates, ${ }^{2}$ the authors conclude that the solubility of aluminium hydroxide in bases is due principally to its colloidal properties, and question the existence of aluminates as definit salts. Since, in a study now in progress to determin the conditions for the quantitative precipitation of aluminium hydroxide, results were obtained which indicated the existence of definit aluminates, a statement of these results, together with a discussion of the conclusions reached by Mahin and his co-workers seems desirable.

In the work at this Bureau the changes in hydrogen ion concentration taking place in a solution during the precipitation and re-solution of aluminium hydroxide by alkalis were determined by means of a hydrogen electrode, using an apparatus and method similar to that employed by Hildebrand and Harned ${ }^{3}$ in the determination of magnesia in limestone. The method used here was briefly as follows: The solution to be titrated was maintained at about $25^{\circ}$ in a beaker which was closed with a rubber stopper having suitable perforations for $(a)$ the hydrogen electrode, $(b)$ the connection with a cell containing saturated potassium chloride solution which was in turn connected to a calomel half-cell in o.r $N$ potassium chloride solution, $(c)$ the tip of the buret, and $(d)$ an exit tube for the hydrogen. By this arrangement carbon dioxide was conveniently expelled from the original solution and excluded during the titration. The readings, made at ten-minute intervals, by means of a millivoltmeter and a capillary electrometer, are probably accurate to \pm 2 millivolts. The aluminium chloride solution, prepared from the recrystallized salt, was about decimolar (for $\mathrm{AlCl}_{3}$ ) and contained some free hydrochloric acid; the sodium and potassium hydroxide solutions prepared from the metals, were about fifth normal. The initial volume of the titration was about $50 \mathrm{cc}$. The details of the method will be described in a later paper, in which the re-

1 Published by permission of the Director of the Bureau of Standards.

2 This Journal, 35, 30-9 (1913).

${ }^{3}$ Orig. Com. 8th Intern. Congr. Appl. Chem., I, 217-25 (1912). Consult also Joel H. Hildebrand, Thrs JourNaL, 35, 864 (rgr3), in which the curve for $\mathrm{Al}_{2}\left(\mathrm{SO}_{4}\right)_{2}+\mathrm{NaOH}$ is shown, and attention called to the formation of $\mathrm{NaAlO}_{2}$. This work though presented on March 25, 1913, in an address at the Milwaukee meeting, was not known to the author till its appearance in the July number of THIS JouRnaI, when the present article was being prepared for press. 